\title{
Healthcare Information Management System in Home Environment
}

\author{
Chang-Sun Shin ${ }^{1}$, Su-Chong Joo ${ }^{2}$, and Chang-Won Jeong ${ }^{2}$ \\ ${ }^{1}$ School of Information and Communication Engineering, Sunchon National University, Korea \\ csshinesunchon.ac. kr \\ ${ }^{2}$ School of Electrical, Electronic and Information Engineering, Wonkwang University, Korea \\ \{scjoo, mediblue\} @wonkwang.ac.kr
}

\begin{abstract}
The distributed object group framework (DOGF) enables easier integration of distributed objects to healthcare home applications. This paper describes a healthcare information management system for supporting healthcare home services based on DOGF. The system architecture consists of healthcare database management tool, framework, sensor manager and physical elements such as sensors, devices and appliances. The focus of this paper is on the design and construction of a database system in a framework to support home healthcare services. Healthcare information is organized using database schemes based on the specific types of data collected from various typedsensors. The database constructed from this information for the purpose of home healthcare services is divided into the base information that uses the real schemes and the context based information that uses the view schemes. To verify the practical use of the healthcare information management system proposed in this paper, we created a prototype healthcare home monitoring service using information, emergency call, and home appliance control. The result of the experimental evaluation shows the comparison of the execution service time of the base information and the context based information in our simulated scenario of a home healthcare application.
\end{abstract}

Keywords: Healthcare Information Management, Distributed Object Group Framework, Healthcare Home Service.

\section{Introduction}

Ubiquitous computing strives to develop application environments able to transparently deal with the mobility and interactions of both users and devices. Current research in ubiquitous computing focuses on building infrastructures for managing active spaces, connecting new devices, or building useful applications to improve functionality [1]. But these researches lack of an integrated management of information which is a very important technology in healthcare field, especially for a ubiquitous home healthcare environment. Since existing healthcare information system is usually constructed independently, there is no interconnection to support total business area such as doctor, nurse, patient and environments. That is, the current focus of healthcare information management is still on patient records. Also, a 
large number of healthcare related applications are available that effectively support specific needs but are isolated or incompatible. Ad hoc solutions to interface various components are expensive and time-consuming because systems use different platforms, programming languages and data formats [2]. In this context the paper introduces the healthcare information management system that supports integrated management of information such as environments, personal health and location based on healthcare home environment. We describe how to design and construct the database scheme for the management of the healthcare information system. Our system is based on the distributed object group framework (DOGF) that enables easy integration of distributed objects to healthcare home applications $[3,4]$. We used the timer-triggered message-triggered Object (TMO) scheme and TMO Support Middleware (TMOSM) for interactions between distributed applications. To verify the practical use of the healthcare information management system proposed in this paper, we present a home healthcare monitoring service prototype consisting of emergency call, appliance control, and so on designed for the living space of an elderly person living alone. Also, we present the experimental results comparing the execution time of the base information and the context based information service.

The rest of the paper is organized as follows. The next section describes the architecture of the healthcare information management system. Section 3 presents the database for healthcare application services based on home environments. Section 4 describes the system procedure and the GUI of the healthcare management tool and sensor manager process for stream data from sensors. We present a prototype of the healthcare home services using the healthcare database and the result of the evaluation. Finally, the last section describes the conclusion and future works.

\section{Healthcare Information Management System}

\subsection{System Architecture}

Our system uses the object group management for domain grouping in DOGF. The needs of a home healthcare environment are considered. The system consists of related services such as location tracking service, healthcare information and supporting service. To facilitate information collection and sharing in this environment, we adopted the TMO scheme and TMOSM [5] into the development environment of the system that we implemented. The architecture of the system is shown in Figure 1 and is organized in five layers. The physical layer contains hardware infrastructures such as various sensors, devices, machines etc. The healthcare database consists of classification of sensor nodes, collected data from the sensors and user profile data including health indicators, service information and access right privileges as information for security, and view information for supporting the service applications. The framework layer contains components of DOGF to support a logical single view of the system environment by grouping them. That is, the group manager API supports the execution of application of appropriate healthcare home services in an upper layer by using the input information obtained from the individual or grouped physical devices through the sensor manger in the lower layer. The tool layer consists of distributed programming developing-tool (DPD-Tool) [6] and healthcare database management tool (HDM-Tool). 


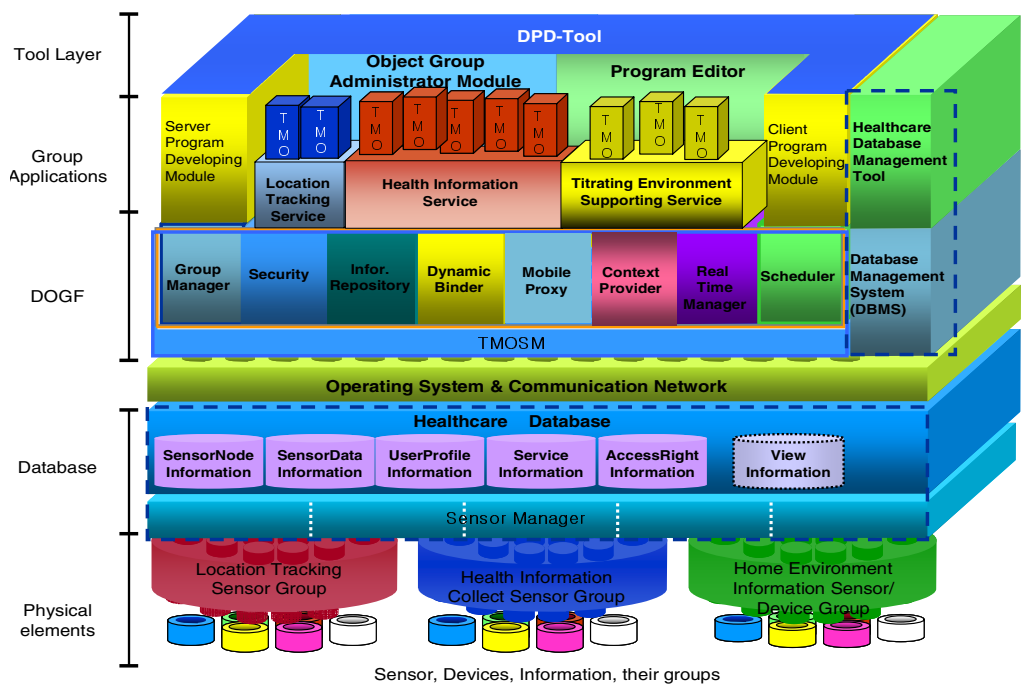

Fig. 1. The architecture for Healthcare Information Management System

\subsection{The Interaction of System Components}

The functionality of the proposed system is illustrated in Figure 1. We defined the interaction of components which include the distributed application, database, sensors and components of framework.

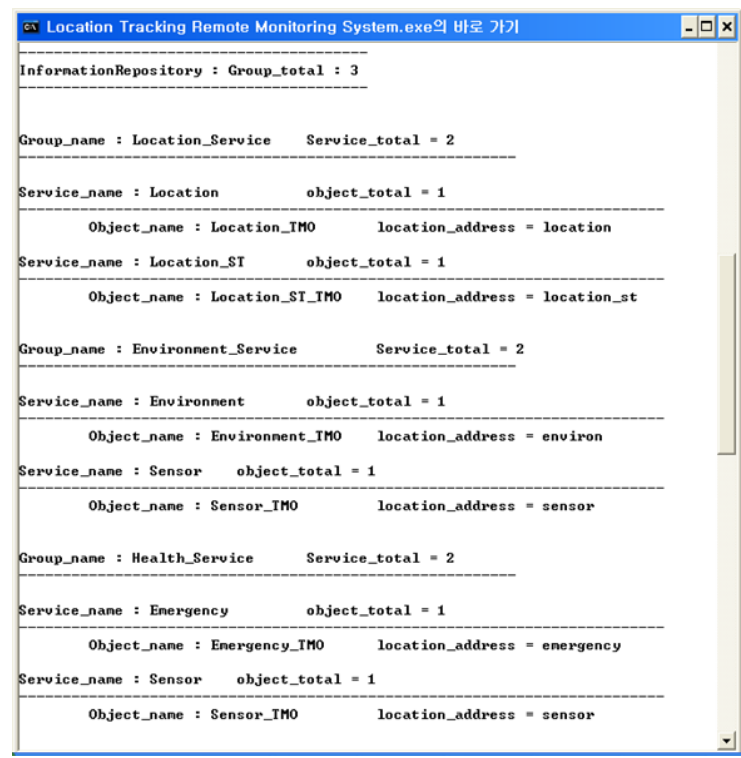

Fig. 2. Process of Grouping Distributed Objects for Healthcare Home Service 
Figure 2 show the process of grouping distributed objects by the group manager object. Also, it provides the interaction of distributed application using APIs and service object references to support collecting real time information from the sensor manager. The security service is provided through security object that handles the access right information of clients through the healthcare database. When a service object is replicated, a dynamic binder object provides the reference of the service object using a binding algorithm. A distributed application obtains real time information from sensor nodes through service object reference which enables a connection to healthcare database. And, the interaction of distributed objects provides the results for the requested service through the framework components.

\section{Healthcare Database}

The healthcare database is constructed by using this information to support the home healthcare services which are classified into the base information which uses real schemes and the context based information which uses view schemes. The base information includes low data obtained from physical sensors which are generally relevant such as location, personal health, environment, and the user profile. And, the context based information that is produced and fused by using the based information. This context based information might be obtained using various view schemes according to the requirements of a particular healthcare application service. In Figure 3 , the base information scheme consists of 14 tables.

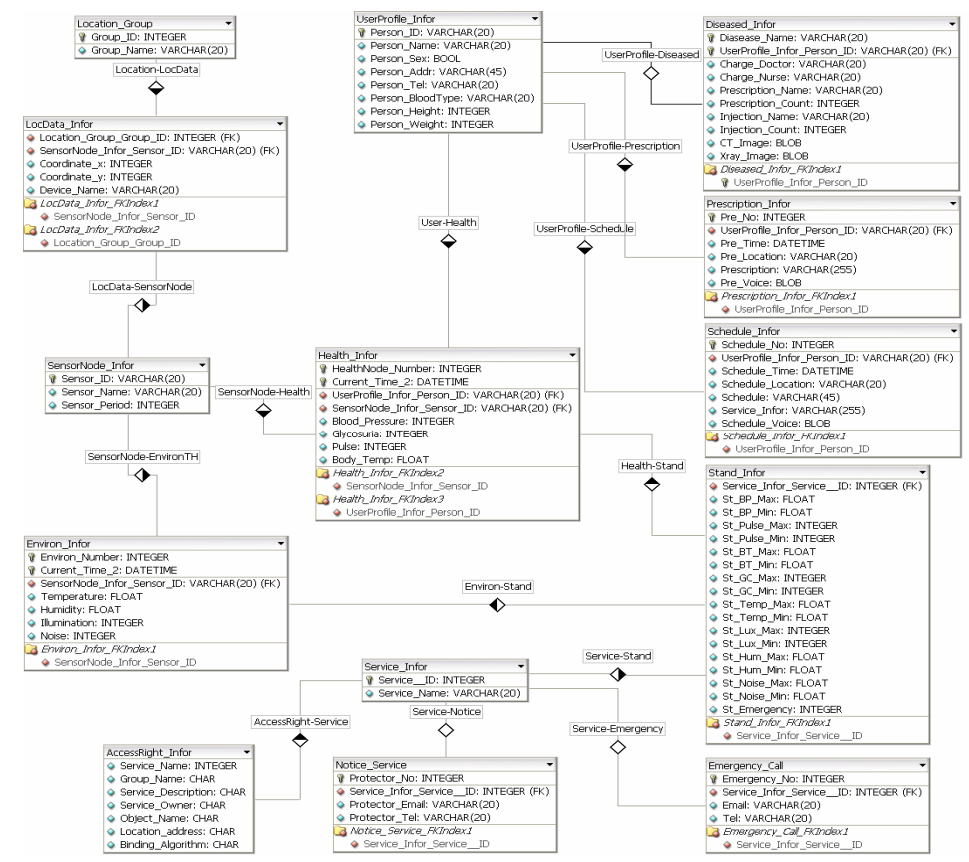

Fig. 3. Healthcare Base Information Scheme 
Figure 4 shows the context based information scheme for healthcare applications. It used the healthcare applications which enable to easily apply the services.

\begin{tabular}{|c|c|}
\hline ApplianceOnOff_Infor & \multirow{4}{*}{$\begin{array}{l}\text { A LocData_Infor.Device_Name: VARCHAR(20) } \\
\text { \& Environ_Infor.All_Data: INTEGER } \\
\text { \& Stand_Infor.All_Data: INTEGER }\end{array}$} \\
\hline T LocData_Infor.Device_Name: VARCHAR(20) & \\
\hline ata: INTEGER & \\
\hline & \\
\hline & \multirow{3}{*}{$\begin{array}{l}\text { Current_State } \\
\text { Q Current_Time_2: DATETIME } \\
\diamond \text { LocData_Infor.Device_Name: VARCHAR(20) } \\
\diamond \text { Current_State: VARCHAR(255) }\end{array}$} \\
\hline HealthInfor_Service & \\
\hline Device_Name: VARCHAR(20) & \\
\hline$\diamond \mathrm{COO}$ & \multirow{4}{*}{$\begin{array}{l}\text { Current_Location } \\
\text { Q Current_Time_2: DATETIME } \\
\& \text { Group_Infor.Group_ID: INTEGER } \\
\diamond \text { LocData_Infor.Device_Name: VARCHAR(20) }\end{array}$} \\
\hline & \\
\hline$\diamond$ Heal & \\
\hline Visiting_Infor & \\
\hline V LocData_Infor.Device_Name: VARCHAR(20) & \\
\hline Group_Infor.Group_ID: INTEGER & Visiting_Count \\
\hline & : INTEGER \\
\hline$\diamond$ Exercise_Quentity: FLOAT & $\diamond$ Location_Count: INTEGER \\
\hline
\end{tabular}

Fig. 4. Healthcare Context Information Scheme

\begin{tabular}{|c|c|}
\hline Moving_Position & Monitoring_Infor \\
\hline V Current_Time_2: DATETIME & \multirow{9}{*}{$\begin{array}{l}\text { \& Current_State: VARCHAR(255) } \\
\text { \& Moving_Position: VARCHAR(255) } \\
\text { \& Quentity_Exercise: VARCHAR(255) } \\
\text { \& ApplianceOnoff_Infor: VARCHAR(255) } \\
\text { \& HealthInfor_Service: VARCHAR(255) } \\
\text { \& Visiting_Count: VARCHAR(255) } \\
\text { \& Notice_Service: VARCHAR(255) } \\
\text { \& Auto_Control: VARCHAR(255) } \\
\text { \& Visiting_Infor: VARCHAR(255) } \\
\text { \& Emergency_Call: VARCHAR(255) }\end{array}$} \\
\hline$\diamond$ Coordinate_x: INTEGER & \\
\hline$\diamond$ Coordinate_y: INTEGER & \\
\hline$\diamond$ Current_Location: VARCHAR(20) & \\
\hline Notice_Service & \\
\hline Service_Count: INTEGER & \\
\hline$\diamond$ Stand_Infor.All_Data: INTEGER & \\
\hline$\diamond$ Health_Infor.All_Data: INTEGER & \\
\hline O Service_Kind: VARCHAR(20) & \\
\hline Quentity_Exercise & Emergency_Call \\
\hline i Current_Time_2: DATETIME & S Service_Count: INTEGER \\
\hline$\diamond$ Moving_Distance: INTEGER & $\diamond$ Group_Infor.Group_ID: INTEGER \\
\hline$\diamond$ Moving_Time: INTEGER & $\diamond$ Stop_Time: INTEGER \\
\hline$\diamond$ Quentity: FLOAT & $\diamond$ Stand_Infor.St_Emergency: INTEGER \\
\hline
\end{tabular}

The context based information constructed using materialized view is saved to service schema and managed using the view scheme. This approach allows server and client program developers to develop healthcare application much faster. The healthcare management tool provides the user interface for managing information directly from healthcare database which is described in the next section.

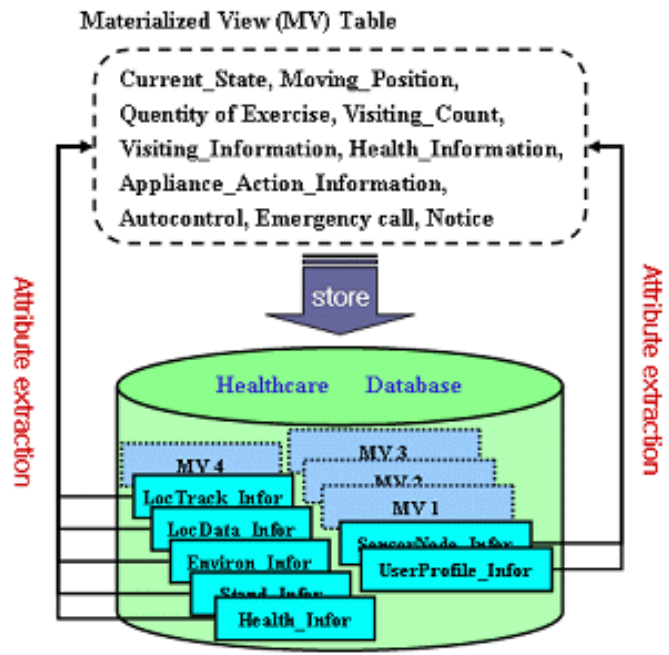

Fig. 5. Context Based Information Using Materialized View Table

\section{Procedure for Healthcare Information Management System}

In this section, we present the procedure of our system which consists of three main processes: 1) collection of healthcare information by the sensor manager, 
2) management of information by the healthcare database management tool and 3) development of a healthcare application.

\subsection{Collection of Healthcare Information}

In order to collect the healthcare information, we developed a sensor manager that is capable of supporting integrated collection of stream data from various sensors. Our sensor manager is similar to a mediator wrapper approach for heterogeneous sensor data management. The sensor manager assumes a role of a local query translator and the query processor for the database. It provides an integrated input stream data process to handle the different types of sensors. The procedures of both stream data process and local query process are described in Figure 6. Figure 7 is the result of stream data process by sensor manager according to the setting information and period information. It includes the received stream data from the sensor, other sensor specific information and collected and processed into an insert query in the database.

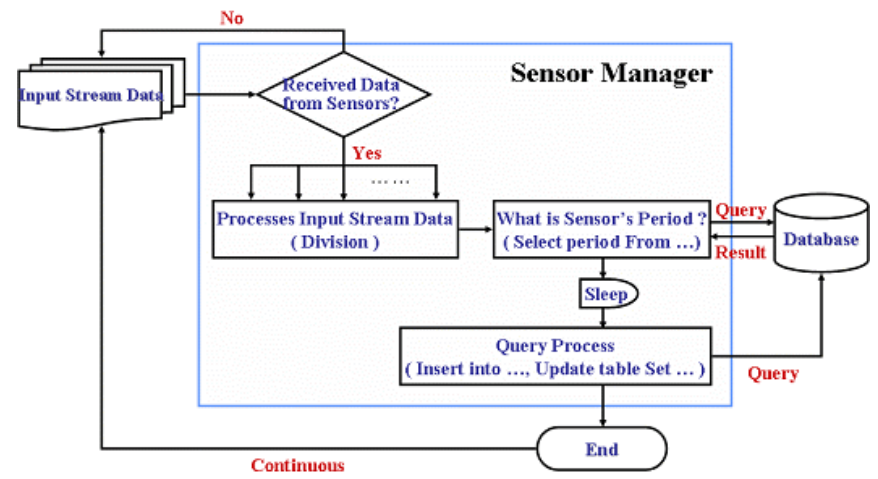

Fig. 6. Context Based Information Using Materialized View Table

(1)

\begin{tabular}{|c|}
\hline ल C:WWINDOWSWSystem 32Wfemd, exe - java SensorManager \\
\hline 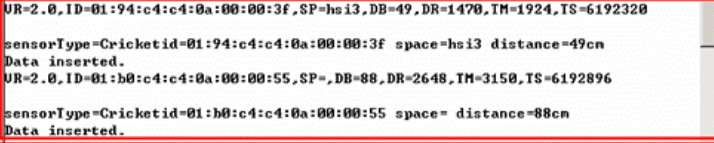 \\
\hline 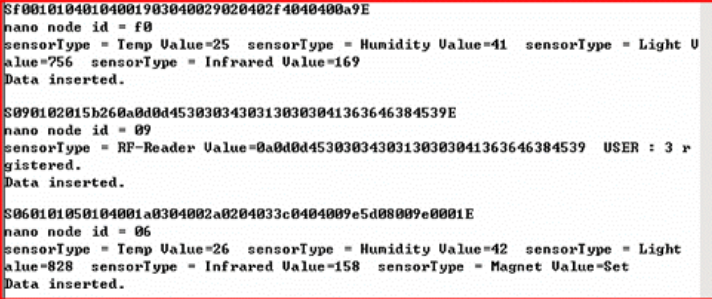 \\
\hline 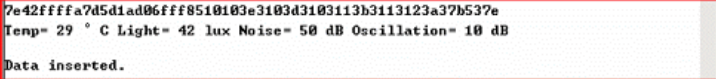 \\
\hline C:HDocuments and Settings Hdskintthy Docunentsthoorks pacettSens \\
\hline
\end{tabular}

Fig. 7. Execute Results of Stream Data Process by Sensor Manager 
Typically, sensors are physically distributed in different locations in different rooms of a building. They are heterogeneous in various ways to handle the diverse types of data such as temperature, localization, luminosity; they have various capacities of computing and storage such as different rates of data delivery (e.g. two measurements per second, a measurement by occurrence of an event) etc. In the current work, we classify according to period of different rates of data delivery. The period information of sensor is managed by the database which controls one or more sensor stream data. The associated methods are matched with the specific to a sensor stream data to enable easier manipulation.

\subsection{Management of Healthcare Information}

The healthcare database management tool (HDMT) handles the management of the healthcare information through an interface to the healthcare database. This includes the database creation and management tasks such as to display the DB List and table data, the query execution by SQL and search of plan by user, write SQL, dictionary information management, and so on. In addition, it provides the functions to handle context based information to generate the view tables. We implemented the user interface using a GUI-window screening panel according to these functions. The panel consists of five-tabbed display with for Table, Query, DB information and Context based information for management of healthcare database.

Figure 8 shows the execution screens for database management in HDMT. The figure shows are results corresponding to given commands such as user written SQL statements, table properties and interface buttons. Data from various sources must be

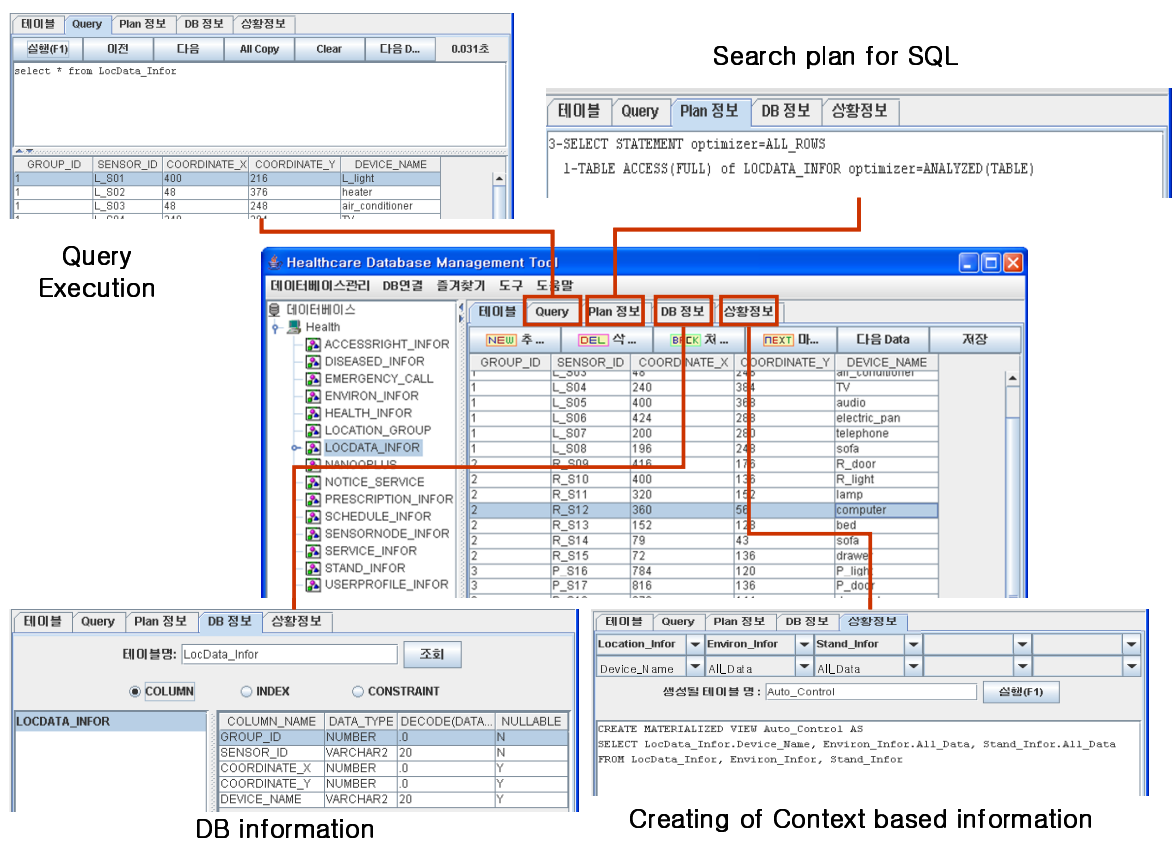

Fig. 8. GUI for Healthcare Database Management Tool 
stored in and retrieved from DBMS, because it must always be possible to use data on the basis of diverse criteria. HDMT manages the collected information using ordinary DBMS and provides the upper layers convenient means to access it.

\subsection{Executing Results of Healthcare Home Service}

To verify the practical use of the proposed system, we show a prototype of a home healthcare monitoring service using information (classified as either base or context based information), emergency call, home appliance control. In this section we describe the results of our evaluation. Figure 9 shows the relationship of the distributed applications with TMO objects and healthcare database.

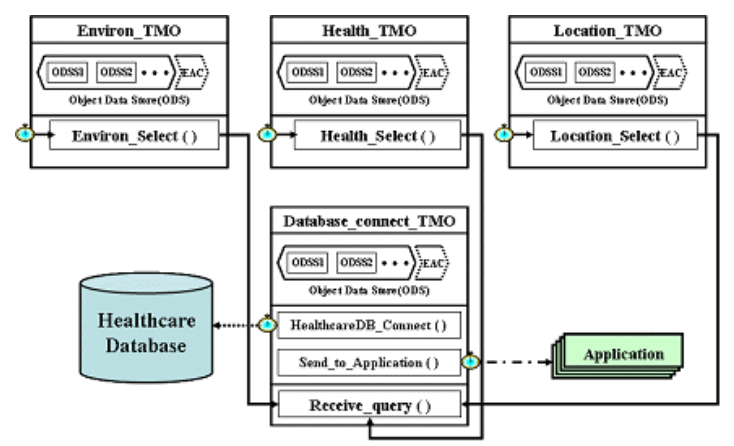

Fig. 9. Interaction of TMOs, Healthcare Database, Applications

Each TMO is assigned a specific role in the healthcare database. The Environ_TMO requests the environment information in healthcare database via Database_connect_TMO. The Database_connect_TMO handles the requested messages of each TMO and sends the result value to application. In the same manner, the Health_TMO, the Location_TMO also have a same function for requesting functions to healthcare database.

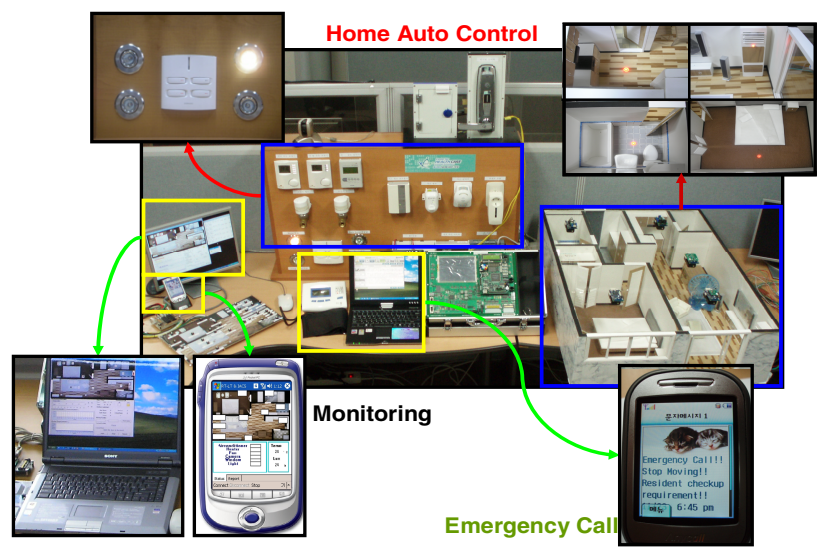

Fig. 10. Physical Environment for Healthcare Home Services 
Figure 10 shows the physical environments for healthcare home services reflecting a real world scenario. It is based on elements shown in Figure 9. From these environments, we verify the functionality of the healthcare home services we designed and constructed. The home healthcare services are provided through home healthcare monitoring devices which enable location tracking, health and environment information for home resident, emergency call as SMS using cell phone and home appliance control based on health, location, standard information specified by user for environments of home such as fan, light, air-conditioner, etc.

We analyzed the service time within which the base information and the context based information is able to process a request and reply with the result in the home healthcare services. Figure 11 shows the average service time.

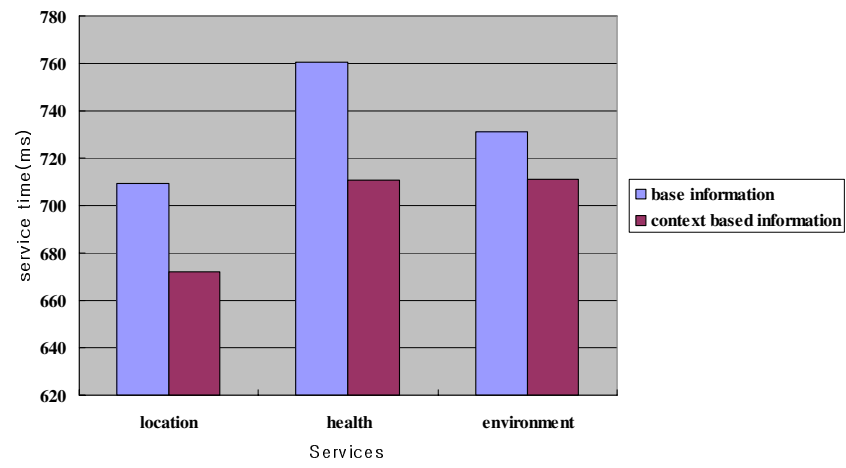

Fig. 11. Average Service Time

The result obtained from this experiment in shown in the graph. For location service the service time difference is $37.4 \mathrm{~ms}$; for the health information service, it is $49.5 \mathrm{~ms}$; and for home auto control service it is $20.3 \mathrm{~ms}$. This evaluation result shows that the context based information could provide a more responsive service for home healthcare.

\section{Conclusions and Future Works}

Existing healthcare information systems are constructed independently without considering integrated management. The current focus of healthcare information management is still on patient records. A large number of healthcare related applications effectively support specific needs but are isolated or incompatible. And interfaces are expensive and time-consuming because systems use different platforms, programming languages and data formats. To solve this problem we proposed a healthcare information management system. Our research focused on the design of healthcare information management system and the development of a health database based on the framework for supporting home healthcare service. We verified that healthcare related information supporting healthcare home services and the context 
based information showed better performance than base information at providing the home healthcare services.

Our future work will focus on the applications of different environments for ubiquitous healthcare service and on improving the functionality of our system. We will also address the relevant security issues in the context of this work.

Acknowledgments. This research was supported by the MIC(Ministry of Information and Communication), Korea, under the ITRC(Information Technology Research Center) support program supervised by the IITA(Institute of Information Technology Assessment)" (IITA-2006-(C1090-0603-0047)).

\section{References}

1. Rodriguez, M., Favela, J., Gonzalez, V., Munoz, M.: Agent Based Mobile Collaboration and Information Access in a Healthcare Environment. In: Proceedings of Workshop of EHealth: Applications of Computing Science in Medicine and Health Care, Cuernavaca, Mexico, pp. 970-936 (December 2003) ISBN: 970-36-0118-9

2. Spyrou, S.S., Bamidis, P., Chouvarda, I., Gogou, G., Tryfon, S.M., Maglaveras, N.: Healthcare information standards: comparison of the approaches. Health Informatics Journal 8, 14-19 (2002)

3. Shin, C.-S., Jeong, C.-W., Joo, S.-C.: Construction of Distributed Object Group Framework and Its Execution Analysis Using Distributed Application Simulation. In: Yang, L.T., Guo, M., Gao, G.R., Jha, N.K. (eds.) EUC 2004. LNCS, vol. 3207, pp. 724-733. Springer, Heidelberg (2004)

4. Shin, C.-S., Lee, C.-S., Joo, S.-C.: Healthcare Home service System Based on Distributed Object Group Framework. In: Gavrilova, M., Gervasi, O., Kumar, V., Tan, C.J.K., Taniar, D., Laganà, A., Mun, Y., Choo, H. (eds.) ICCSA 2006. LNCS, vol. 3983, pp. 798-807. Springer, Heidelberg (2006)

5. Kim, K.H., Ishida, M., Liu, J.: An Efficient Middleware Architecture Supporting Timetriggered Message-triggered Objects and an NT-based Implementation. In: ISORC'99. Proceedings of the IEEE CS 2nd International Symposium on Object-oriented Real-time distributed Computing, pp. 54-63. IEEE Computer Society Press, Los Alamitos (1999)

6. Jeong, C.-W., Kim, D.-S., Lee, G.-Y., Joo, S.-C.: Distributed Programming Developing Tool Based on Distributed Object Group Framework. In: Gavrilova, M., Gervasi, O., Kumar, V., Tan, C.J.K., Taniar, D., Laganà, A., Mun, Y., Choo, H. (eds.) ICCSA 2006. LNCS, vol. 3983, pp. 853-863. Springer, Heidelberg (2006) 